\title{
Observation of elastic anisotropy in strained optical nanofibers using Brillouin spectroscopy
}

\author{
Adrien Godet, Jacques Chrétien, Thibaut Sylvestre, Jean-Charles Beugnot, Kien Phan Huy \\ Institut FEMTO-ST, Université de Bourgogne Franche-Comté, CNRS, UMR6174, Besançon, France
}

Optical nanofibers (ONFs) are excellent nanophotonic platforms for many applications such as optical sensing, quantum photonics, and nonlinear optics, due to both tight optical confinement and their evanescent field. From an acoustic viewpoint, it has recently been reported the observation of a new class of Brillouin acoustic resonances in optical nanofibers, including hybrid shear/longitudinal acoustic waves (HAWs) and surface acoustic waves (SAWs) [1-2]. It has been later shown that, under axial tensile strain, the Brillouin frequency shifts (BFS) of these elastic resonances are fundamentally different from that of standard optical fibers [3]. This is principally due to the hybrid nature of acoustic waves and thus classical theory used for standard fibers can no longer be used [4]. Here, we develop a theoretical model based on third-order elasticity of silica to predict the strain dependence of acoustic waves in ONFs. We show in particular that the fundamental elastic properties of silica dramatically change due to elastic anisotropy and transverse hardening. The agreement with experimental results is excellent.

Figure 1(a) shows a scheme of the tapered optical fiber under test including the uniform nanofiber section with a diameter of $660 \mathrm{~nm}$ and a length of $80 \mathrm{~mm}$. The backward Brillouin spectrum was measured by using heterodyne detection and the tensile strains were applied by stretching the ONF using translation stages, as shown in the bottom of Fig. 1(a). The Brillouin spectrum of the unstrained fiber taper is shown in red in Fig.1 (b). We can note several peaks in the GHz range due to HAWs, SAWs, and the untapered fiber (SMF), as indicated by black arrows [1]. Tensile strain up to 5\% in elongation was then applied on the ONF and the results are plotted in Fig. 1(c) as a colormap. We can clearly see a continuous frequency shift of all the Brillouin resonances (red lines) with different slopes from $98 \mathrm{MHz} / \%$ for SAWs up to $350 \mathrm{MHz} / \%$ for HAWs. We also note the crossing of the two SAWs around $5 \%$.
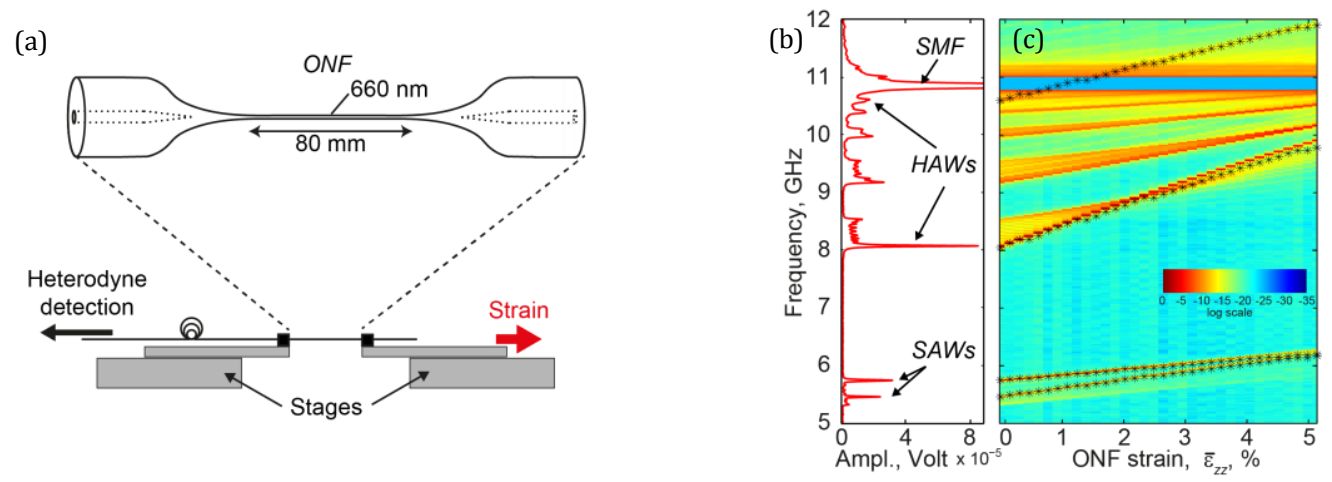

Fig. 1 (a) Top: Scheme of a tapered optical fiber with a uniform nanofiber section. Bottom: Experimental setup to apply tensile strain and to measure the Brillouin backscattering spectrum. (b) Brillouin spectrum of the unstrained tapered fiber. The black arrows show the surface and hybrid acoustic resonances. (c) Experimental (red lines) and theoretical (black crosses) results of Brillouin spectrum as a function of tensile strain.

We developed a theoretical model to predict these unexpected BFS variations. It is based on third-order decomposition of the elastic tensor $c^{\prime}$ which, in the case of subwavelength optical fiber, is highly dependent on the axial strain $\bar{\epsilon}_{z z}$ and expressed by the following equation, $c^{\prime}\left(\bar{\epsilon}_{z z}\right)=c_{i j k l}+c_{i j k l z z} \bar{\epsilon}_{z z}$, where $c_{i j k l}$ and $c_{i j k l z z}$ are the second and third-order elastic tensors of bulk silica [5]. $c$ ' has actually 5 different coefficients, instead of 3 in isotropic media, which dramatically changes the elastic properties of silica. The tensor thus acquires a strong anisotropy with off-diagonal coefficients that strongly affect all acoustic waves. The problem is then solved using the elastodynamic equation including the variation of elastic constants $c^{\prime}\left(\bar{\epsilon}_{z z}\right)$ with strain. The result of the model is shown in Fig.1 (c) as black crosses on the experimental data. An excellent agreement is found between experimental and theoretical Brillouin frequency shifts for all resonances. We checked that this model is valid for any nanofiber diameter. This result opens up new applications for point sensors, in particular for the discrimination between the effect of strain and temperature.

\section{References}

[1] J.C. Beugnot et al., "Brillouin light scattering from surface acoustic waves in a subwavelength-diameter optical fiber," Nat. Commun, 5:5242 (2014).

[2] A. Godet et al., "Brillouin spectroscopy of optical microfibers and nanofibers," Optica 4, 1232-1238 (2017).

[3] A. Godet et al., "Tensile strain dependence of Brillouin scattering in tapered optical fibers," The European Conference on Lasers and Electro-Optics. Optical Society of America (2017).

[4] T. Horiguchi et al., "Tensile Strain Dependence of Brillouin Frequency Shift in Silica Optical Fibers", IEEE Phot. Tech. Lett. 1, 107 (1989).

[5] E. H. Bogardus, "Third-Order Elastic Constants of Ge, MgO, and Fused SiO2," Journal of Applied Physics 36, 2504-2513 (1965). 